\title{
PRECURSORS AND GENETIC CONTROL OF PIGMENTATION
}

\section{HYDROXYLATION AND METHOXYLATION STAGES IN ANTHOCYANIDIN SYNTHESIS}

\author{
B. J. HARRISON and R. G. STICKLAND \\ John Innes Institute, Colney Lane, Norwich NR4 7UH
}

Received 10.v.77

\section{SUMmary}

Induced anthocyanidin synthesis in various genotypes of acyanic Antirrhinum majus flowers after the imbibition of flower extracts from a range of species producing more complex anthocyanidins (delphinidin, peonidin, petunidin and capensidin) enabled an analysis of the stages at which hydroxylation and methoxylation occurred in the donor plant. Methylation and the addition of the $5^{\prime}$ hydroxyl appear to occur at a late stage in the biosynthetic pathway but the interfeeding of Antirrhinum genotypes showed an early hydroxylation up to the dihydroxy stage. Biosynthesis of a glycoside of tamarixetinidin was demonstrated for the first time.

\section{Introduction}

THE biosynthetic pathway to anthocyanidin production in Antirrhinum majus (fig. 1) can be genetically blocked at three major stages: (a) pre-flavanone by nivea (niv); (b) pre-flavanonol and post-flavanone by incolorata (inc); and (c) pre-anthocyanidin and post-flavanonol by some pallida (pal) alleles and (in the corolla tube only) by delila (Geissman, et al., 1954; Fincham and Harrison, 1967). More complex anthocyanidins contain a variety of

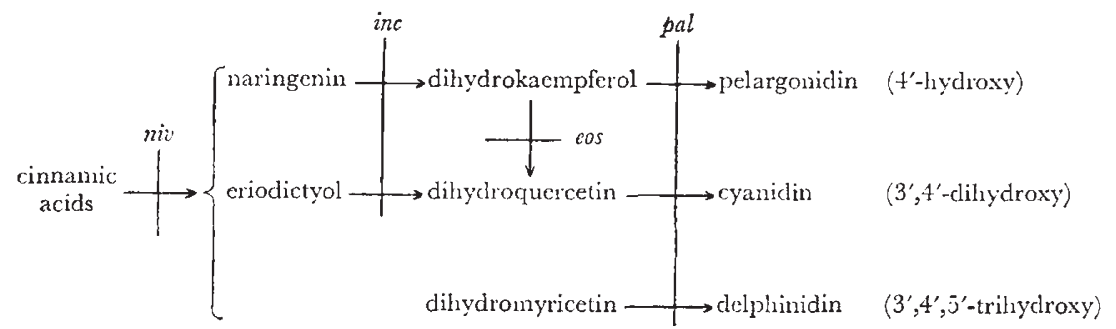

Frg. 1

hydroxyl and methoxyl groups which must be added to the basic molecular structure at some stage in the pathway. At present there are two possibilities: either that relatively simple anthocyanidins are formed first and that subsequently these have more hydroxyl or methyl groups added; or, as suggested by Hess (1967), that the complex hydroxyl/methoxyl pattern is laid down at the cinnamic acid stage (before the flavonoid skeleton is formed) and that this pattern persists through the biosynthetic sequence to produce the corresponding anthocyanidin. In the first case the precursors would be relatively simple compounds; in the second case, in flowers producing 
complex anthocyanidins, the precursors would be equally complex. If these natural precursors were fed to A. majus flowers, it should be possible to determine their nature from the anthocyanidins formed.

It has been shown (Harrison and Stickland, 1974; Stickland and Harrison, 1974) that extra hydroxyl/methoxyl elaboration can occur in several plant species tested; however, if the precursor or homogenate donor is elaborated beyond the genetic potential of the recipient flower, then no reduction in the degree of hydroxylation or methoxylation occurs. Thus, naringenin and dihydrokaempferol (4'-hydroxy) can each initiate production of pelargonidin* (4'-hydroxy) in an A. majus of eosinea (eos): niv genotype and cyanidin (3', 4'-dihydroxy) in an Eos: niv flower. (The eos/Eos gene controls the normal pelargonidin or cyanidin production, and extra hydroxyl being added to the introduced precursor by action of the Eos allele). If eriodictyol or dihydroquercetin $\left(3^{\prime}, 4^{\prime}\right.$-dihydroxy) is administered to similar genotypes, only cyanidin can be synthesised; no removal of the superfluous hydroxyl occurs in the eos/eos genotype. Therefore, the eos/eos and Eos genotypes of A. majus, if blocked for anthocyanidin production (for example by niv), enable a screening of precursors for their hydroxylation and methoxylation configuration even when the donor homogenate is obtained from other genera. The Eos Antirrhinum not only contains an enzyme that can add an extra hydroxyl group to produce cyanidin from 4'-hydroxyl precursors (e.g. naringenin or dihydrokaempferol) but in addition these flowers are capable of forming anthocyanidins from precursors found in flowers from other species. The $A$. majus enzymes can act on precursors with an extra hydroxyl to form from dihydromyricetin $\left(3^{\prime}, 4^{\prime}, 5^{\prime}\right.$-trihydroxy), an anthocyanidin delphinidin not normally found in this Antirrhinum species.

\section{MATERIALS AND METHODS}

Donor homogenates were prepared by grinding the fresh flowers in liquid nitrogen and the precursors were extracted by ethyl acetate and administered as an aqueous solution to the recipient flowers by the immersion of the corolla tubes. Each recipient flower was fed an extract prepared from about 20 times its weight of donor flowers.

The inc Eos, niv:eos, niv:Eos and pal-tincta lines of Antirrhinum majus were inbred lines maintained at this Institute as standard genetic stocks. The Petunia hybrida material was selected from a commercial line of mixed types.

Induced pigments were extracted from the recipient flowers by immersion in cold $\left(2^{\circ} \mathrm{C}\right)$ methanol containing 1 per cent $(\mathrm{w} / \mathrm{v}) \mathrm{HCl}$ for $1-2$ days. Analytical procedures followed the methods of Harborne (1967) except that thin-layer chromatography on cellulose was employed. Solvents were butan-1-ol: acetic acid: water $(4: 0 \cdot 6: 5, \mathrm{BAW})$, butan-1-ol: $2 \mathrm{~N} \mathrm{HCl}$ (1: $1, \mathrm{BuHCl})$, acetic acid: conc. $\mathrm{HCl}:$ water $(15: 3: 82, \mathrm{HOAc}-\mathrm{HCl})$, acetic acid: conc. $\mathrm{HCl}$ : water $(30: 3: 10$, Forestal) and 1 per cent HCl.

The amount of induced anthocyanidin varied considerably with the age of the flower and was normally in the range of 20-50 n moles per flower. As little as $3 \mathrm{n}$ moles of minor anthocyanidins should have been detectable. Only qualitative assessments were made in the present study but quantitative

* For clarity glycosidic forms are not specified but designated simply as the anthocyanidins. 
analysis under controlled conditions showed that the amount of synthesised cyanidin in inc/inc Antirrhinum majus flowers increased from 10 to $250 \mathrm{n}$ moles/g wet wt. as the dihydroquercetin concentration was raised from $0 \cdot 3$ to $10 \mathrm{~mm}$ (Stickland, Harrison, and Barnes, 1976).

Spectral shifts with $\mathrm{AlCl}_{3}$ were obtained using reagents dried over aluminosilicate molecular seive and this resulted in larger shifts than normally recorded (see table 2).

\section{Results and conclusions}

The precursors extracted from an acyanic Primula obconica in which delphinidin was present as pseudo-base (a colourless form which gives rise to normal anthocyanidin on treatment with dilute acid in the cold) were imbibed by inc A. majus flowers. Only cyanidin was formed in both the eos/eos and Eos genotypes (table 1), which suggests that the Primula contained dihydroquercetin (3', 4'-dihydroxy). Had the Primula extract contained dihydrokaempferol (4'-hydroxy) then pelargonidin would have been synthesised in the eos/eos genotype; however, if dihydromyricetin ( $3^{\prime}, 4^{\prime}$, $5^{\prime}$-trihydroxy) was present then the corresponding anthocyanidin delphinidin would have been produced in both $A$. majus genotypes.

TABLE I

Induced hydroxylation structures

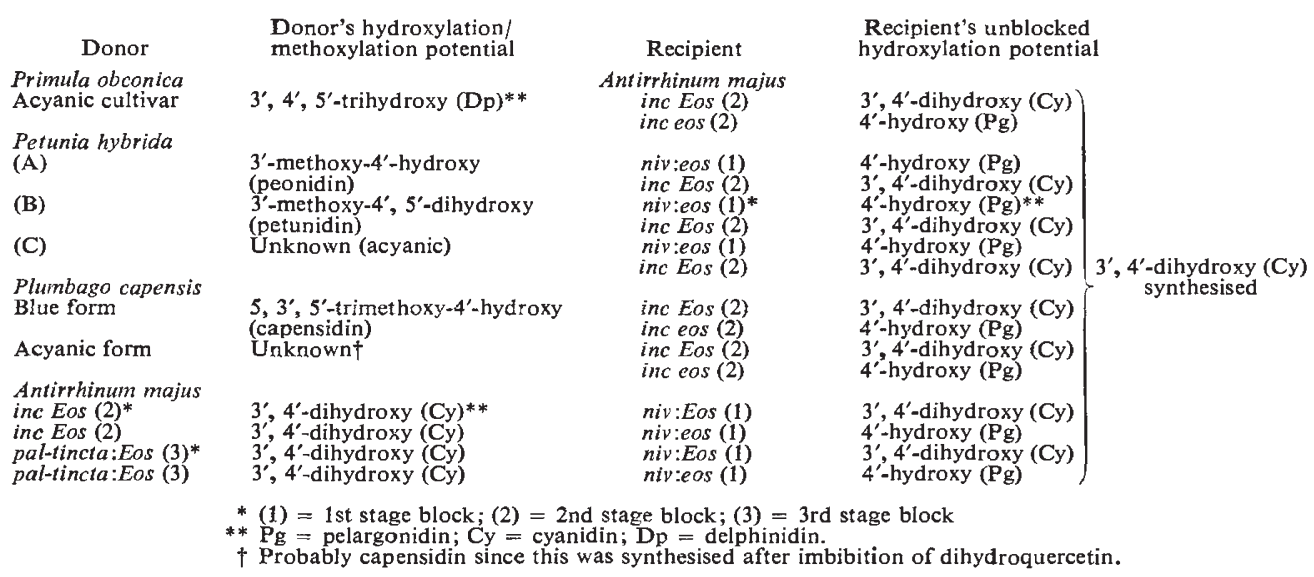

Similarly, precursors were extracted from the flowers of three Petunia hybrida varieties: (A) containing peonidin $\left(3^{\prime}\right.$-methoxy-4'-hydroxy) as the only flower pigment, (B) containing petunidin ( $3^{\prime}$-methoxy-4', $5^{\prime}$-dihydroxy) as the only flower pigment, and (C) a white form, and also from the blue flowers of Plumbago capensis (containing capensidin, 5, 3', 5'-tri-O-methyldelphinidin, as the sole anthocyanidin) and from the white form of the same species. In all five cases, the extracts induced the synthesis only of cyanidin when fed to $A$. majus (table 1). A. majus has been shown to synthesise anthocyanidins with greater hydroxyl/methoxyl elaboration than pelargonidin and cyanidin but induced synthesis of petunidin, peonidin and

40/1-I 
capensidin has not yet been tried because the corresponding precursors were unavailable.

A more complicated situation arose from extracts of the flowers of the "Dazzler" variety of Primula sinensis. It is known that these flowers contain pelargonidin, cyanidin and peonidin and also dihydrokaempferol (Harborne and Sherratt, 1961). When a "Dazzler" extract was fed to an eos A. majus, pelargonidin and cyanidin were produced. These anthocyanidins were presumably formed from the corresponding precursors dihydrokaempferol and dihydroquercetin; however, no peonidin was formed in the $A$. majus flowers, suggesting an absence of the appropriate methylated precursor. That the $A$. majus enzymes are capable of using methylated precursors was shown by feeding hesperitin (which has a $3^{\prime}$-hydroxy-4'-methoxy substitution pattern) to niv:eos or niv:Eos flowers. A small amount of anthocyanin was formed which was slightly redder in colour than the normal cyanidin-3rutinoside and differed from it in $R_{F}$ values in four solvents (table 2). Hydrolysis in $\mathrm{N} \mathrm{HCl}$ showed the unknown anthocyanin to be a diglycoside and the aglycone had $R_{F}$ values differing from those of cyanidin. Absence of a spectral shift with $\mathrm{AlCl}_{3}$ indicated a $3^{\prime}$ - or 4'-methoxy group. From the available evidence the unknown anthocyanin could have been peonidin-3rutinoside ( $3^{\prime}$-methoxy) but since it was formed from hesperitin it would appear more likely that it was the 4 '-methoxy compound for which we suggest the name tamarixetinidin-3-rutinoside from the corresponding flavonol tamarixetin.

TABle 2

Data on unknown anthocyanin formed from hesperitin

\begin{tabular}{|c|c|c|c|c|c|}
\hline & BAW & $\mathrm{BuHCl}$ & $\begin{array}{c}R_{F} \text { value } \\
(\times 100) \text { in } \\
1 \% \mathrm{HCl}\end{array}$ & $\mathrm{HOAc} / \mathrm{HCl}$ & Forestal \\
\hline Cy†-3-rutinoside & $36(37)^{*}$ & $27(25)$ & $12(19)$ & $35(43)$ & -- \\
\hline Pn†-3-rutinoside & $-(34)$ & $-(14)$ & $-(16)$ & $-(41)$ & $-\ldots$ \\
\hline Unknown anthocyanin & $29-$ & $21-$ & $9-$ & $31-$ & $-\ldots$ \\
\hline Cyanidin & $45(68)$ & -- & $\ldots$ & - - - & $51(49)$ \\
\hline Peonidin & $-(71)$ & $-\ldots$ & -- & -- & $-(63)$ \\
\hline Unknown aglycone & $47-$ & - - & $-\ldots$ & $-\ldots$ & $66-$ \\
\hline
\end{tabular}

Progressive hydrolysis products

min. in $\mathrm{N}$. $\mathrm{HCl}$
at $100^{\circ} \mathrm{C}$

0 and 3

3 and 10

10 and 30
$R_{F}$
Cy-3-rutinoside 35
Cy-3-glucoside 29
Cy

$F$ values $(\times 100)$ in BAW

$\begin{array}{ll}\text { Unknown diglycoside } & 28 \\ \text { Unknown monoglycoside } & 19 \\ \text { Unknown aglycone } & 47\end{array}$

Absorption maxima $(\mathrm{nm})$ in $0.01 \% \mathrm{HCl}$ in $\mathrm{MeOH}$

\begin{tabular}{|c|c|c|c|c|}
\hline & & & & $\begin{array}{c}\text { Spectral } \\
\text { shift with } \\
\mathrm{AlCl}_{3}\end{array}$ \\
\hline Cy-3-rutinoside & $524(523)$ & Cyanidin & $534(535)$ & 41 (18) \\
\hline Pn-3-rutinoside & $-(523)$ & Peonidin & $-(532)$ & $-(0)$ \\
\hline Unknown anthocyanin & $523-$ & Unknown aglycone & $532-$ & $0-$ \\
\hline
\end{tabular}

* Figures in bracket are those quoted by Harborne (1967) and the $R_{F}$ values are for paper chromatography.

$f \mathrm{Cy}=$ cyanidin; $\mathrm{Pn}=$ peonidin. 
These experiments show that, although the Primula, Petunia and Plumbago flowers contained anthocyanidins more complex than cyanidin, only cyanidin was produced when extracts were fed to $A$. majus even though the recipient A. majus flowers were capable of forming methylated anthocyanidins (as tamarixetinidin above). Similarly, anthocyanidins with more elaborate hydroxylation than cyanidin have been produced in $A$. majus following the imbibition of dihydromyricetin to produce delphinidin (Stickland and Harrison, 1974).

It would thus seem that at least in some material at the dihydroflavonol stage the main anthocyanidin precursor in these flowers is no more complex than dihydroquercetin $\left(3^{\prime}, 4^{\prime}\right.$-dihydroxy) and that the $5^{\prime}$-hydroxyl group and any methyl groups are added at a very late stage in the biosynthetic pathway, although the possibility of traces of other precursors being present cannot be completely eliminated.

The biosynthetic route may be different in Endymion species (Stickland and Harrison, 1977) since white forms capable of forming anthocyanidin from dihydroquercetin produced cyanidin and not the delphinidin usually present in the blue flowered types. This means either that all these white forms were acyanic mutants of the rare magenta (cyanidin containing) type, or more probably that in the normal blue flowers the delphinidin is formed via dihydromyricetin rather than from dihydroquercetin and that in these flowers no mechanism exists for the conversion of dihydroquercetin to delphinidin. However, it has been shown that dihydroquercetin can be elaborated to $3^{\prime}, 4^{\prime}, 5^{\prime}$-trisubstituted anthocyanidins in the following white flowered plants: Streptocarpus (Maassen's White) $\rightarrow$ malvidin; Petunia hybrida $\rightarrow$ malvidin; Primula obconica $\rightarrow$ delphinidin; and, Catharanthus roseus $\rightarrow$ hirsutinidin. Attempts to induce synthesis by feeding extracts of blue flowered Endymion non-scriptus to $A$. majus genotypes were unsuccessful; this suggests there was no unused precursor (dihydromyricetin or otherwise) present, for delphinidin has been synthesised in $A$. majus from this intermediate (Stickland and Harrison, 1974).

In contrast to the situation concerning $5^{\prime}$-hydroxylation and methylation is that of $3^{\prime}$-hydroxy or $3^{\prime}, 4^{\prime}$-dihydroxy substitution. If Eos A. majus flowers blocked either at the inc middle stage or at the late pal-tincta stage in synthesis were fed as extracts separately to niv:eos flowers then cyanidin was produced, showing that the donor plants contain eriodictyol and dihydroquercetin respectively. Hydroxylation up to this $3^{\prime}, 4^{\prime}$-dihydroxy stage therefore occurs early, at least in this particular pathway.

\section{References}

fincham, J. R. S., AND HARrison, B. J. 1967. Instability at the Pal locus in Antirrhinum majus. (2) Multiple alleles produced by mutation of one original unstable allele. Heredity, 22, 211-224.

geissman, T. A., JORGensen, E. C., AnD JOHnSON, B. L. 1954. The chemistry of flower pigmentation in Antirrhinum majus. Color genotypes. I. The flavonoid components of the homozygous P,M,Y color types. Archs Biochem. Biophys., 49, 368-388.

harborne, J. B. 1967. Comparative Biochemistry of the Flavonoids. Academic Press.

HARboRne, J. B., AND SHERRATT, H. S. A. 1961. Plant polyphenols. 3. Flavonoids in genotypes of Primula sinensis. Biochem. 7., 78, 298-306.

HARRISON, B. J., AND STICKLAND, R. G. 1974. Precursors and genetic control of pigmentation. 2. Genotype analysis of pigment controlling genes in acyanic phenotypes in Antirrhinum majus. Heredity, 33, 112-115. 
HEss, D. 1967. Die Wirkung von Zimtsauren auf die Arthocyansynthese in isolierten Petalen von Petunia hybrida. Z. Pfanzenphysiol, 56, 12-19.

STIGKLAND, R. G., AND HARRISON, B. J. 1974. Precursors and genetic control of pigmentation. 1. Induced biosynthesis of pelargonidin, cyanidin and delphinidin in Antirrhinum majus. Heredity, 33, 108-112.

STICKLAND, R. G., AND HARRISON, B. J. 1977. Precursors and genetic control of pigmentation. 3. Detection and distribution of different white genotypes of bluebells (Endymion species). Heredity, 39, 327-333.

STIGKLAND, R. G., HARRISON, B. J., AND BARNEs, G. 1976. Quantitative effects of partial dominance and optical activity of precursors on anthocyanidin production. Fohn Innes 67th Annual Report. 